\title{
Ultraviolet-Induced Flashover of a Plastic Insulator Using a Pulsed Excimer Laser
}

\author{
C. L. Enloe ${ }^{1}$ and R. M. Gilgenbach ${ }^{1}$
}

Received July 10, 1986; revised September 11, 1986

\begin{abstract}
Ultraviolet-induced flashover has been observed over stressed, angled, acrylic insulators illuminated by a short (60 ns) pulse of excimer laser light at $249 \mathrm{~nm}$. Flashover has been observed at ultraviolet fluences of $5-65 \mathrm{~mJ} / \mathrm{cm}^{2}$ for electric field stresses approximately $10-30 \%$ of static breakdown stress. Insulators at positive angle (conventional configuration) exhibit a reduced tolerance to ultraviolet light versus insulators at negative angle (unconventional configuration) by approximately a factor of 2 , while the presence of impurities at the triple point reduces the tolerance to ultraviolet even further. Flashover is related to the fluence, rather than the power density, for short pulses, and the production of photoelectrons is a likely mechanism for the initiation of flashover.
\end{abstract}

KEY WORDS: Insulator flashover; ultraviolet; surface plasma.

\section{INTRODUCTION}

It has long been known that the interface of a solid dielectric insulator with vacuum is one of the weak points of a high-voltage transmission system, because an insulator in vacuum is subject to flashover at high electric field stress. Researchers in the field of high-energy-density plasmas have found that intense ultraviolet radiation can initiate the flashover process at the dielectric/vacuum interface. ${ }^{(1,2)}$ Subsequent bench tests have shown that the phenomenon exists even at relatively low field stress (approximately $10 \%$ of self-breakdown stress). ${ }^{(3,4)}$ While ultraviolet-induced insulator flashover is a bane to designers of pulsed-power systems, it may well be a boon in the development of low-inductance, high-current, triggerable surfacetracking switches, ${ }^{(5,6)}$ large-area flashboards for ion diodes, in plasma processing applications where it is desirable to create a large-area uniform surface discharge plasma in a controlled manner, or as new xuv light sources. With the recent development of multi-microsecond electron beam

'Intense Energy Beam Interaction Laboratory, Department of Nuclear Engineering, University of Michigan, Ann Arbor, Michigan 48109. 
accelerators such as the Michigan Electron Long-Beam Accelerator ${ }^{(7)}$ (MELBA) there exists increased concern about the performance of the insulator stacks which may be subjected to intense ultraviolet radiation.

One drawback with previous ultraviolet-induced flashover experiments was that, although it was apparent that the moderate to hard portion of the ultraviolet spectrum $(h \nu \geq 4 \mathrm{eV})$ was responsible for initiating flashover, it was unclear exactly what the characteristics of the illumination were in this region. Pinched plasma devices were previously used as illumination sources; consequently the illumination was broadband and irregular in time, usually appearing in several discrete pulses of many microseconds duration. The difficulties associated with interpreting such data have been eliminated in this experiment by using an ultraviolet excimer laser as the illumination source. The illumination is monochromatic and is applied in a single, near-gaussian pulse. The laser has the added advantage that the portion of the insulator which is illuminated can be closely controlled.

\section{EXPERIMENTAL APPARATUS}

The experimental apparatus is illustrated in Fig. 1. It consists of an illumination source and its associated optics, a test chamber in which a sample insulator is placed, and a high-voltage supply with a voltage monitor.
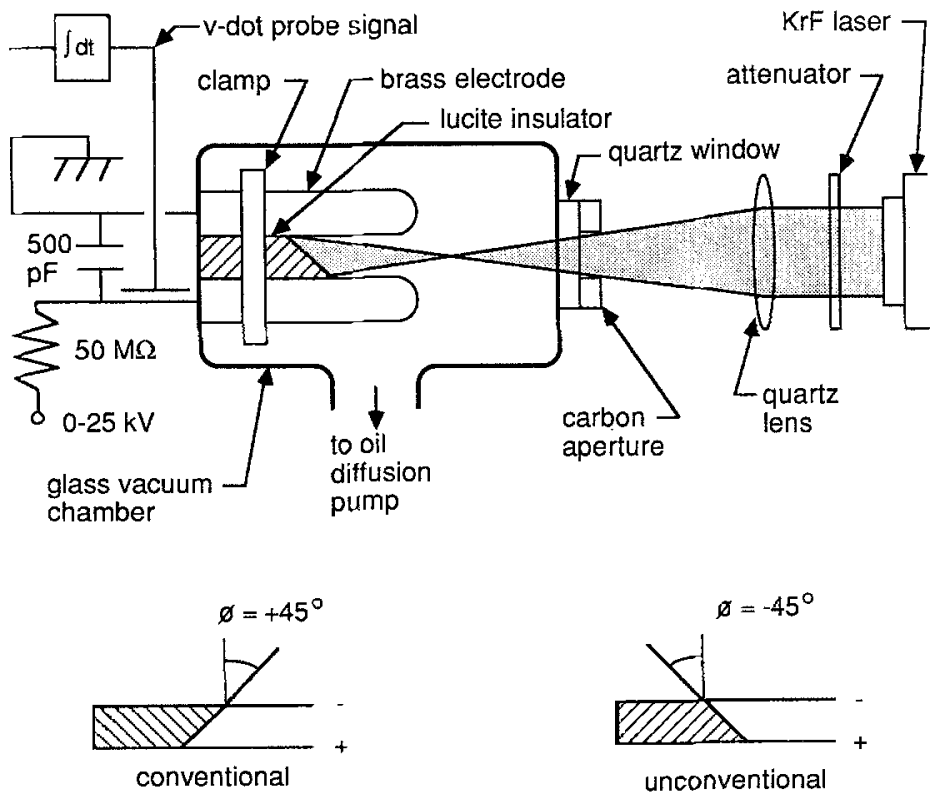

Fig. 1. Experimental apparatus for investigating ultraviolet-induced insulator flashover. 
Ultraviolet illumination was provided by a krypton fluoride excimer laser (Lumonics TE-292) at $249 \mathrm{~nm}(h \nu=5 \mathrm{eV})$. The laser emits a uniform rectangular beam, approximately $2.5 \mathrm{~cm}$ high $\times 3.0 \mathrm{~cm}$ wide at the laser aperture. The beam was imaged using a simple planoconvex quartz lens onto a $1.1-\mathrm{cm}^{2}$ profile at the insulator surface. Thus the entire output of the laser was used to illuminate a small portion of the insulator surface including the anode and cathode triple points, while the electrodes remained unilluminated except for a small area at the triple point itself which was in the falling edge of laser illumination. A carbon aperture was used to eliminate stray light on the electrodes, and this aperture could be varied to further restrict illumination of the insulator surface.

The temporal pulse shape of the laser was measured by an ultravioletsensitive PIN diode and is shown in Fig. 2. The pulse has a full width at half maximum of $27 \pm 2 \mathrm{~ns}$ and a full width at the base of $58 \pm 2 \mathrm{~ns}$ where the small variation is indicative both of shot-to-shot differences and differences between various fills of the lasing gas mixture. A large-area calorimeter was used to monitor the total laser energy. From the pulse shape and the energy deposited, the peak intensity could be calculated. The beam was attenuated using filters made of thin sheets of polyethylene stretched on a metal frame.

A quartz window admitted the laser light to a Pyrex vacuum chamber evacuated to typically $5-6 \times 10^{-5}$ Torr by a 2 -inch oil diffusion pump. The insulator sample was made of $0.63 \mathrm{~cm}$ acrylic stock approximately $10 \mathrm{~cm}$ wide. This material was chosen because it is the material used on MELBA and many other similar devices. The exposed surface was angled at $45^{\circ}$

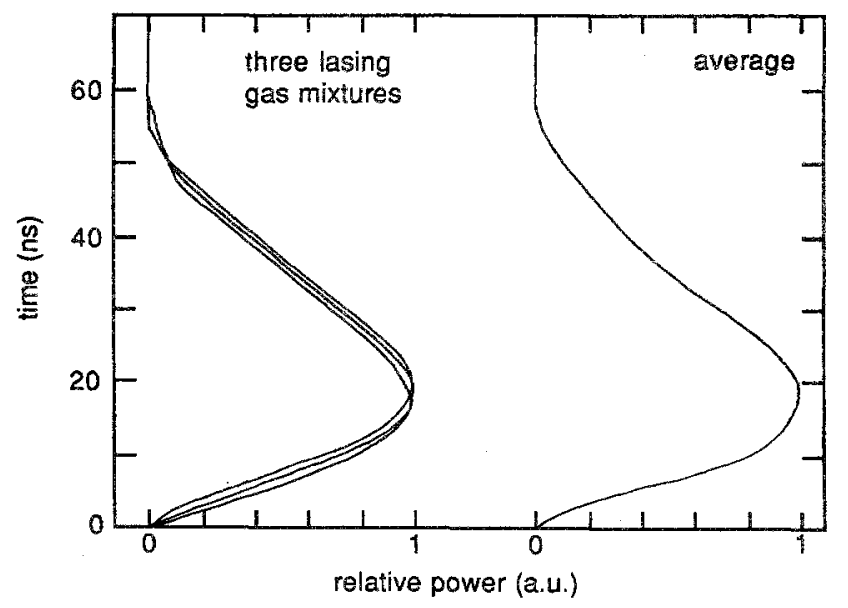

Fig. 2. Laser pulse shape. 
according to typical practice ${ }^{(8-10)}$ and was smoothed with 600 -grit carbide paper. Data are presented with the insulator installed in both a positive angle (conventional) and a negative angle (unconventional) configuration, as indicated in Fig. 1. The insulator was held between two brass electrodes, which extended forward well beyond the insulator surface to eliminate irregularities due to fringing fields in the gap. An insulating clamp was placed around the electrodes to ensure that good contact was made between the electrodes and the insulator.

High D.C. voltage was applied between the electrodes while the voltage was monitored with a passively integrated $\mathrm{V}$-dot probe in an oil tank external to the vacuum chamber. Voltage was maintained with a small $(500 \mathrm{pF})$ capacitor isolated from the power supply by a large ( $50 \mathrm{M} \Omega)$ resistor. A maximum voltage $V=25 \mathrm{kV}$ could be obtained, for a maximum electric field in the gap of $E=39.3 \mathrm{kV} / \mathrm{cm}$. The flashover strength of acrylic reported in the literature is approximately $200 \mathrm{kV} / \mathrm{cm}$ for both configurations ${ }^{(8)}$; the insulator was far from static breakdown. Nevertheless, flashover was observed under ultraviolet illumination. Because the energy in the capacitor was small, the flashover produced no noticeable damage to the insulator surface, and many shots could be taken before cleaning or replacing the insulator sample. Timing between the laser PIN diode signal and the voltage probe was carefully measured to allow timing resolution to within $\pm 2 \mathrm{~ns}$.

Open-shutter photography was used, viewing along the edge of the insulator to obtain a profile of the discharge, or viewing obliquely to determine the portion of the insulator over which flashover occurred.

\section{EXPERIMENTAL PROCEDURE}

In a typical shot series, the insulator sample and the electrodes were cleaned with methanol, the insulator was clamped in place, and the system was evacuated. The initial energy in the unattenuated laser pulse was measured. High voltage was applied, the laser was pulsed to illuminate the insulator, and the voltage probe indicated flashover by a collapse of voltage across the electrodes. The time at which flashover occurred was measured. Attenuation was added to reduce the laser intensity on the insulator surface and another shot was taken. As the laser intensity was decreased, flashover occurred later in time. Further attenuation was added and shots were taken until the voltage probe indicated that flashover was no longer induced. At this point, the voltage was varied and the procedure repeated. Voltage was varied randomly to avoid any conditioning effects or systematic errors. After approximately 20 shots the insulator was removed, examined, and cleaned. 
Care was taken to ensure that the laser illumination was well characterized. The laser energy tended to decrease monotonically after a large number of shots since the fluorine in the lasing mix reacted with the electrodes in the lasing chamber. By monitoring the laser energy frequently between shots on the insulator, the energy in the unattenuated beam could be predicted at any time. Periodically, burn patterns were taken on developed, unexposed Polaroid film. When the illumination began to appear uneven (after approximately 50 shots) the lasing gas mixture was replaced so that the effect of hot spots in the laser illumination was avoided.

\section{EXPERIMENTAL DATA AND ANALYSIS}

Data from a single shot is shown in Fig. 3. We identify several times from this figure. We take the time $t=0$ to be the start of the laser pulse. The laser intensity peaks at $t_{\mathrm{p}}$, where $t_{\mathrm{p}}=20 \pm 2 \mathrm{~ns}$. We take the time at which flashover occurs, $t_{\mathrm{f}}$, to be the beginning of the rapid ( $\leq 2 \mathrm{~ns}$ ) collapse of voltage across the electrodes. For the data presented in this paper $10 \mathrm{~ns} \leq t_{\mathrm{f}} \leq 70 \mathrm{~ns}$. In some data, there is a prebreakdown voltage droop, indicative of a small prebreakdown current in the gap. We define the width of this droop as $w$. For these data $0 \leq w \leq 8 \mathrm{~ns}$. The width $w$ tended to zero at high electric fields.

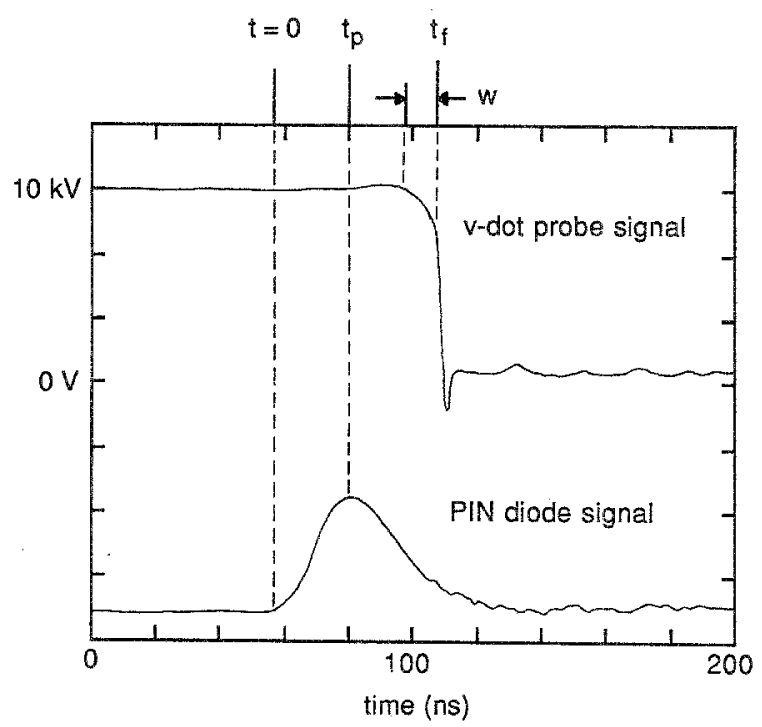

Fig. 3. Sample data: voltage across the electrodes (top) and laser illumination (bottom) versus time. 
The laser pulse shape is constant for all data, so that we need only to know the peak intensity $I_{\max }$ to characterize the intensity $I(t)$ for the entire pulse. Clearly, laser light which illuminates the gap after flashover occurs has no role in the process that initiates flashover. (After a low-impedance path is created, the dynamics of the plasma in the gap will be dominated by the external circuit.) Therefore, we may define the fluence $F$ as

$$
F \equiv \int_{0}^{t_{f}} I(t) d t
$$

One basic question which needs to be addressed is whether ultravioletinduced insulator flashover is dependent upon ultraviolet intensity $\left(\mathrm{W} / \mathrm{cm}^{2}\right)$ or total fluence $\left(\mathrm{J} / \mathrm{cm}^{2}\right)$ at the time of flashover. Therefore we present the data as time to flash versus peak intensity (a) and fluence (b) for several values of the electric field $E$ in Figs. 4-8. The time to flash is dependent on the details of $I(t)$ and is specific to this illumination source. However, as the intensity is varied by a factor of $2-6$ for the various configurations, the fluence at the time of flashover is nearly constant. Further, the mere fact that the flashover is observed much later than the peak of $I(t)$ argues that ultraviolet-induced flashover is a function of fluence. If flashover were initiated at a particular critical intensity, that intensity would always be attained, if at all, on the rising edge of the pulse, so that $t_{\mathrm{f}} \leq t_{\mathrm{p}}$. But in fact, the data show that in many cases $t_{\mathrm{f}}>t_{\mathrm{p}}$ by as much as $40 \mathrm{~ns}$. The question of formative time-lag may be eliminated, because the data show that the prebreakdown phenomena occur on a much shorter time scale than this $(0 \leq w \leq 8 \mathrm{~ns})$, while flashover itself forms quite rapidly ( $\leq 2 \mathrm{~ns})$. Therefore
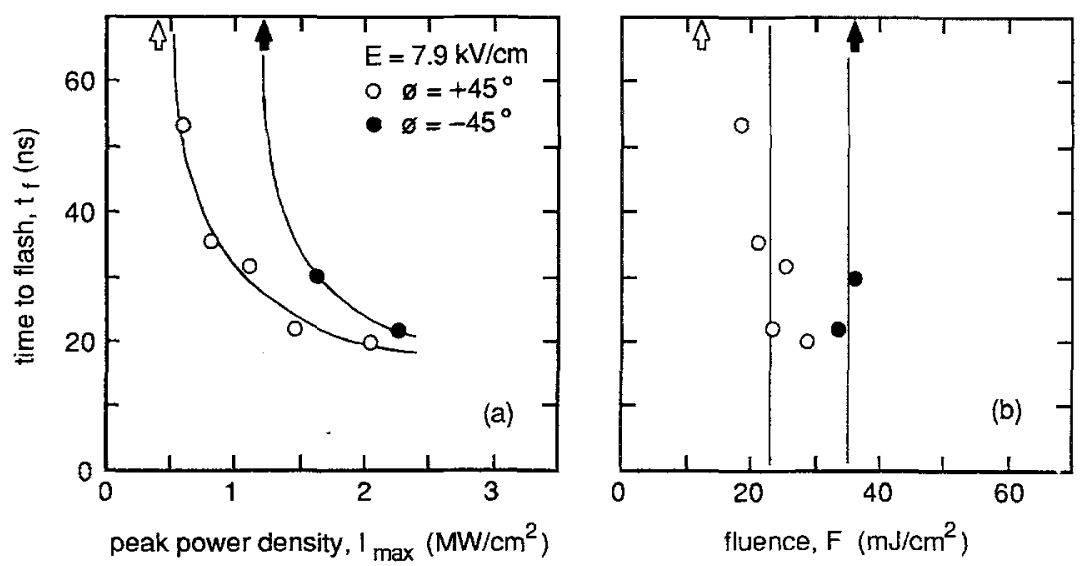

Fig. 4. Flashover behavior for insulators under ultraviolet illumination for electric field stress of $7.9 \mathrm{kV} / \mathrm{cm}$. Arrows indicate shots for which flashover was not observed. 

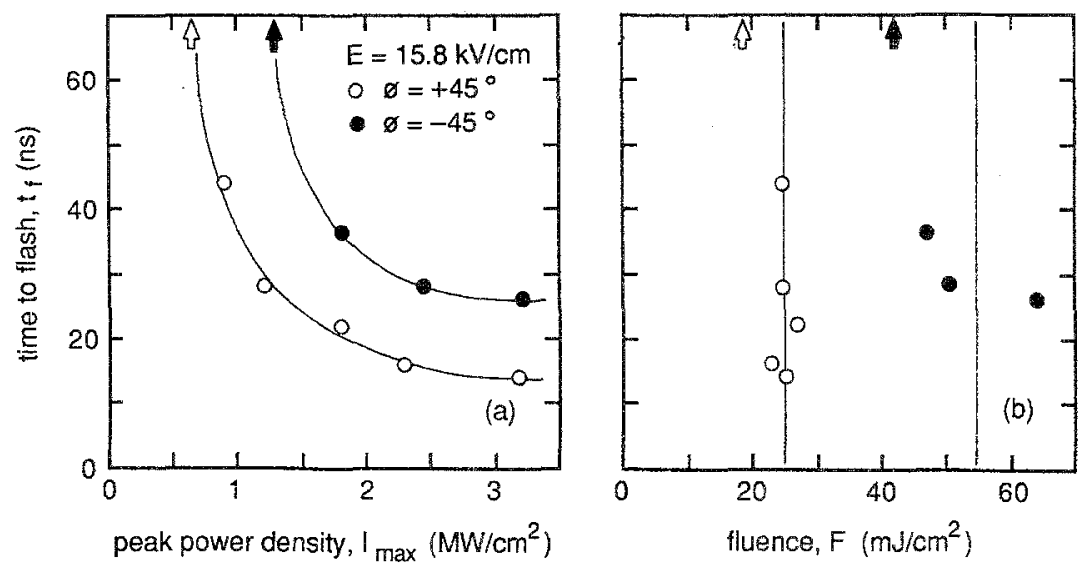

Fig. 5. Flashover behavior for insulators under ultraviolet illumination for electric field stress of $15.8 \mathrm{kV} / \mathrm{cm}$.

we must conclude that it is fluence rather than intensity which is the key in ultraviolet-induced flashover. The data show that the critical fluence to initiate flashover is independent of $I(t)$ for this wavelength.

On the other hand, the critical fluence does depend on the electric field stress and the geometry. Figures 4-7 show the significant result that the unconventional geometry is more resistant to ultraviolet-induced flashover by up to a factor of 2 in fluence. Figure 9 compiles the data shown in Figs. 4-8 for both geometries, displaying the fluences required to initiate fiashover shown in the right part of each figure versus electric field. Although there
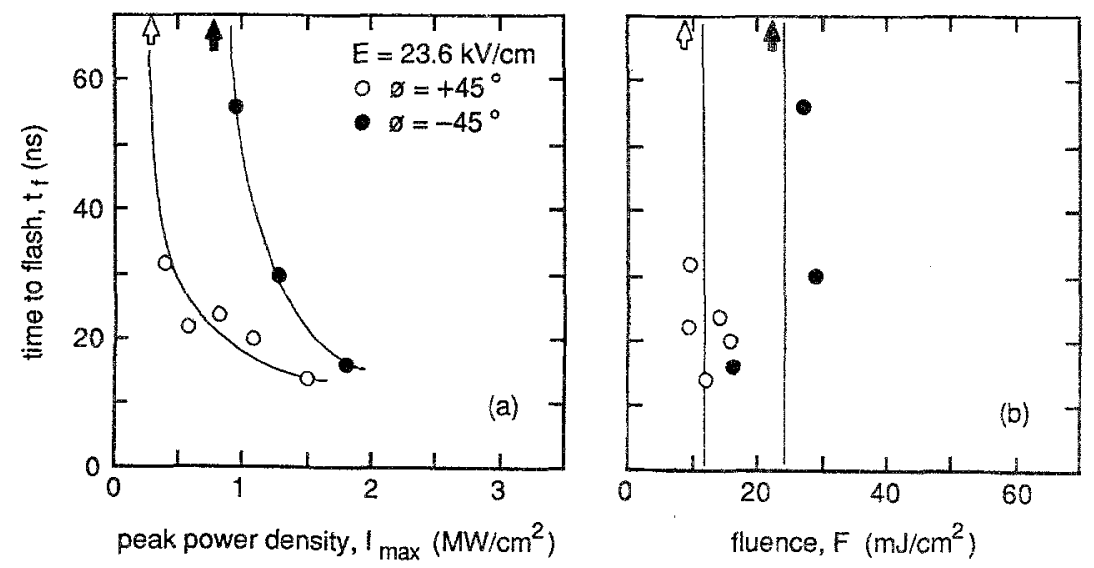

Fig. 6. Flashover behavior for insulators under ultraviolet illumination for electric field stress of $23.6 \mathrm{kV} / \mathrm{cm}$. 

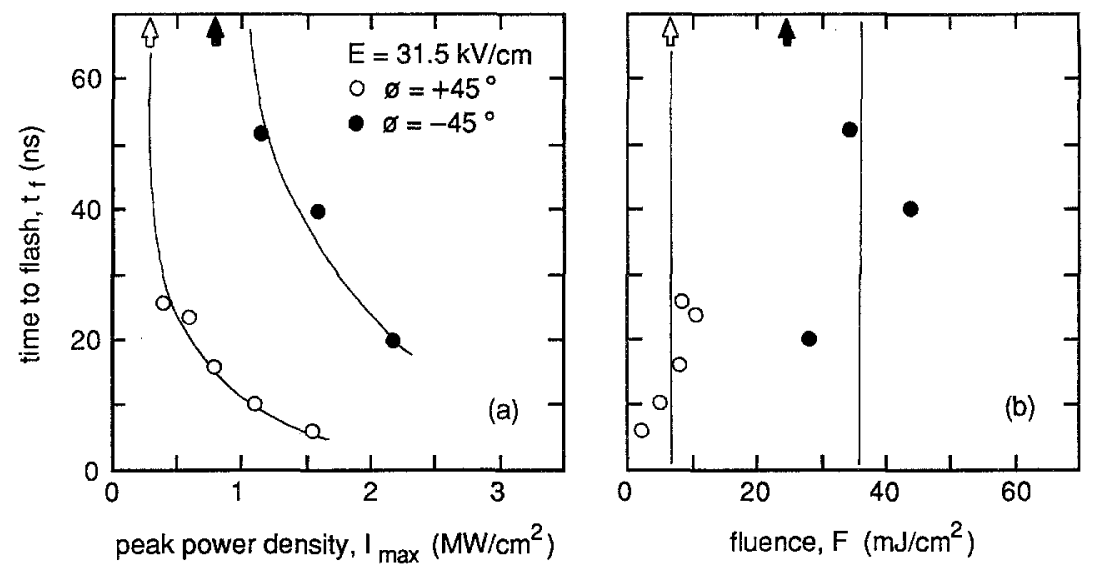

Fig. 7. Flashover behavior for insulators under ultraviolet illumination for electric field stress of $31.5 \mathrm{kV} / \mathrm{cm}$.

is scatter in the data (typical of electrical breakdown phenomena), two trends are clear: (1) flashover voltage decreases as the ultraviolet fluence increases, and (2) the unconventional insulator orientation is more tolerant to ultraviolet illumination than the conventional. This latter observation is consistent with previous ultraviolet-induced insulator flashover studies. ${ }^{(3-4)}$

Open-shutter photography, as shown in Fig. 10, reveals that in the positive-angle configuration (lower photo) the discharge connects with the cathode triple point (as indicated by the bright spot in the photograph at that point) but appears to miss the anode triple point. In the negative-angle
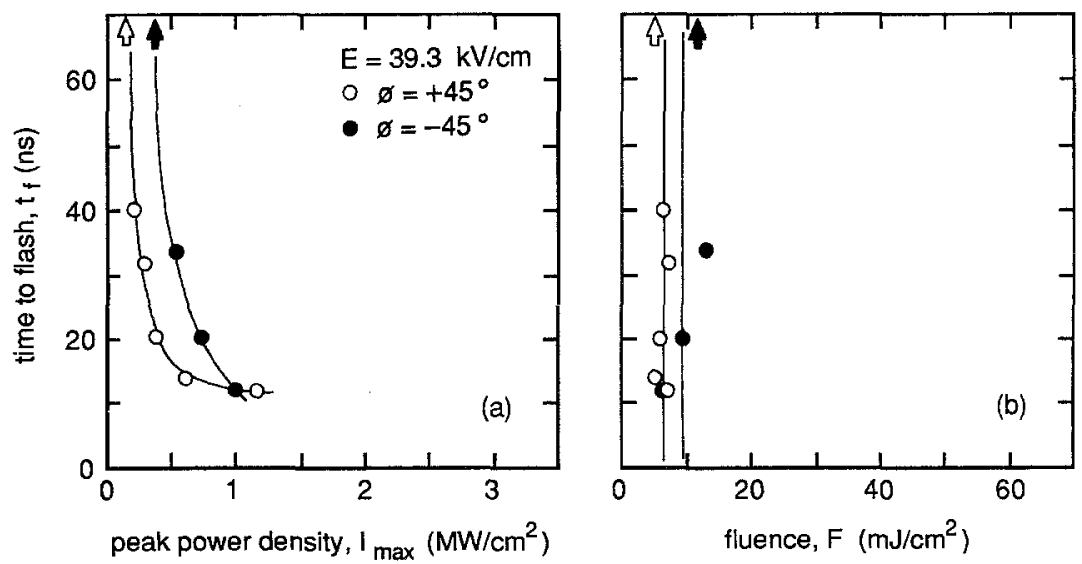

Fig. 8. Flashover behavior for insulators under ultraviolet illumination for electric field stress of $39.3 \mathrm{kV} / \mathrm{cm}$. 


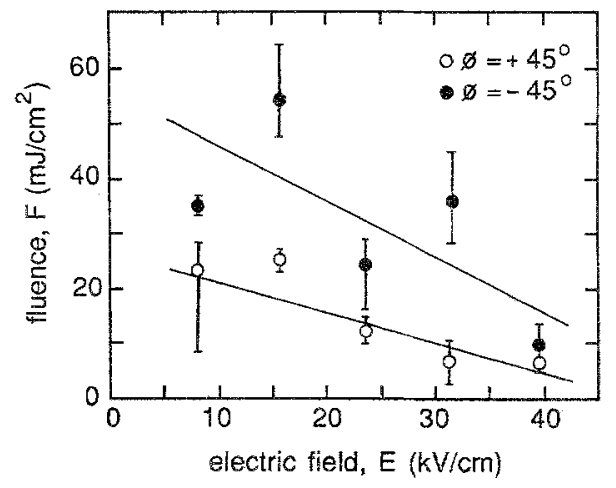

Fig. 9. Fluence required to initiate flashover as a function of electric field stress. Lines represent a least-squares fit to all data for which breakdown was observed.

configuration (upper photo), the opposite appears to be true: the discharge connects with the anode triple point, but misses the cathode triple point. The discharge remains generally near the insulator surface in the negativeangle case, but appears to lift off a portion of the surface in the positive angle case. This lifting-off is more pronounced at higher voltages.

\section{DISCUSSION}

The flashover behavior of insulators in vacuum under pulsed overm voltages has been well characterized. ${ }^{(8,11-13)}$ The systematic investigation of insulator flashover under intense ultraviolet illumination has only begun over the last few years, ${ }^{(3,4)}$ so that detailed models do not yet exist. Given
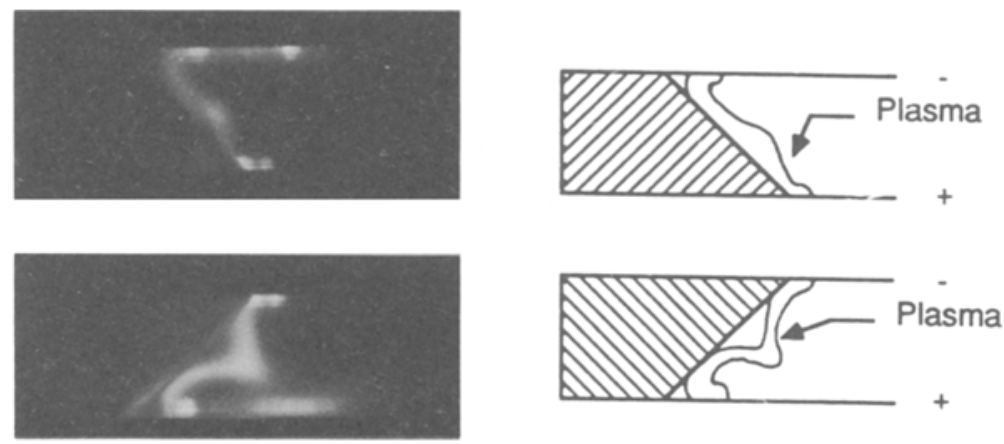

Fig. 10. Open-shutter photographs of insulator flashover at an electric field of $31.5 \mathrm{kV} / \mathrm{cm}$ for: insulators at positive (lower) and negative (upper) angle. 
the short formation times seen in these data, the photoemission of electrons, especially in the high-field regions of the triple point, seems to be the most plausible explanation, versus other mechanisms such as gas desorption from the insulator surface. The question arises, if illumination of the triple point is a key to ultraviolet-induced insulator flashover, can flashover be avoided by blocking illumination of the offending triple point? Our experimental observations indicate that it can. In an early experiment in this series, at low voltage and low power density (no converging optics used) in the positive-angle configuration, flashover was not induced when the cathode triple point was blocked, but flashover was induced when the triple point was illuminated, regardless of what other areas of the insulator were illuminated.

The role of impurities also appears to be important. In one series of shots with the insulator at positive angle, dielectric oil was found to have leaked through the vacuum feed-through and to have been drawn by capillary action up to the cathode triple point, although the insulator surface itself remained clean. Flashover voltage under ultraviolet illumination was found to have been reduced by approximately a factor of 2 versus a clean insulator. Vacuum grease accidentally left in the gap was found to cause a similar lowering of the flashover voltage. Since these contaminants may be present in practical systems, future research must develop a better physical understanding of their role in ultraviolet-induced insulator flashover.

\section{ACKNOWLEDGMENTS}

This research was supported by SDIO-IST, ONR, AFOSR, and a Presidential Young Investigator Award from the NSF. One of the authors (C.L.E.) was supported by AFIT/CI.

\section{REFERENCES}

1. P. J. Turchi and W. L. Baker, J. Appl. Phys. 44, 4936 (1973).

2. W. L. Baker, M. C. Clark, J. H. Degnan, G. F. Kiuttu, C. R. McClenahan, and R. E. Reinovsky, J. Appl. Phys. 49, 4694 (1978).

3. C. L. Enloe, R. Blaher, M. Coffing, and R. E. Reinovsky, Proceedings of the 10th International Symposium on Discharges and Electrical Insulation in Vacuum, Columbia, South Carolina, 1982 (IEEE Catalog No. 82CH1826-7), p. 308.

4. C. L. Enloe and R. E. Reinovsky, Proceedings of the 4th IEEE Pulsed Power Conference, Albuquerque, New Mexico, 1983 (IEEE Catalog No. 83CH1908-3), p. 679.

5. A. H. Guenther and J. R. Bettis, J. Phys. D: Appl. Phys. 11, 1577 (1978).

6. A. H. Guenther and J. R. Bettis, Proceedings of the 5th IEEE Pulsed Power Conference, Arlington, Virginia 1985 (IEEE Catalog No. 85C2121-2), p. 47.

7. R. M. Gilgenbach, Proceedings of the 5th IEEE Pulsed Power Conference Arlington, Virginia, 1985 (IEEE Catalog No. 85C2121-2), p. 126. 
8. O. Milton, IEEE Trans. Electr. Insul. EI-7, 9 (1972).

9. A. Watson, J. Appl. Phys. 38, 2019 (1967).

10. R. Hawley, Vacuum 18, 383 (1968).

11. P. H. Gleichauf, J. Appl. Phys. 22, 766 (1951).

12. E. S. Borovik and B. P. Batrakov, Sov. Phys. Tech. Phys. 3, 1811 (1958).

13. A. A. Avdienko and M. D. Malev, Sov. Phys. Tech. Phys. 22, 986 (1977). 